\title{
Evaluation of Rational Use of Antiretrovirals before the Dolutegravir Transition in Kinshasa, Democratic Republic of Congo
}

\author{
Erick N. Kamangu ${ }^{1,2 *}$, Ben I. Bulanda1, Idriss M. Mwanaut ${ }^{1}$, \\ Simplice K. Makoka' ${ }^{1}$, Gauthier K. Mesia ${ }^{3}$ \\ 1"Focus HIV/AIDS” Research Group, Kinshasa, Democratic Republic of Congo \\ ${ }^{2}$ HIV/AIDS Unit, Service of Molecular Biology, Department of Basic Sciences, Faculty of Medicine, University of Kinshasa, \\ Kinshasa, Democratic Republic of Congo \\ ${ }^{3}$ Unit of Clinical Pharmacology and Pharmacovigilance, Department of Pharmacology and Therapeutics, Faculty of \\ Pharmaceutical Sciences, University of Kinshasa, Kinshasa, Democratic Republic of Congo \\ Email: *erick.kamangu@unikin.ac.cd
}

How to cite this paper: Kamangu, E.N., Bulanda, B.I., Mwanaut, I.M., Makoka, S.K. and Mesia, G.K. (2021) Evaluation of Rational Use of Antiretrovirals before the Dolutegravir Transition in Kinshasa, Democratic Republic of Congo. World Journal of AIDS, 11, 41-49.

https://doi.org/10.4236/wja.2021.112004

Received: January 16, 2021

Accepted: May 5, 2021

Published: May 8, 2021

Copyright $\odot 2021$ by author(s) and Scientific Research Publishing Inc. This work is licensed under the Creative Commons Attribution International License (CC BY 4.0).

http://creativecommons.org/licenses/by/4.0/

\begin{abstract}
Background: The ultimate goal of AntiRetroViral Treatments (ART) is to achieve complete immune restoration and lasting viral suppression in the infected patient. In order to ensure the efficacy, safety and accessibility of antiretroviral drugs (ARVs), it is recommended that they should be prescribed according to national guidelines; which are evolving with the various recommendations of the World Health Organization (WHO) and the arrival of newer, more effective and safer molecules. Objective: The objective of this study was to assess the rational use of Antiretrovirals in patients treated in Kinshasa before the use of Dolutegravir within the national program in order to assess the correct use of these molecules. Methods: This work is a descriptive cross-sectional study to assess the rational use of first-line ARVs among People Living with HIV (PLHIV) in different Centers of Treatment (ATCs) in Kinshasa before the introduction of Dolutegravir. The records of patients on ARVs were randomly and rationally selected in 12 different ATCs for HIV in Kinshasa according to three centers per district in the period from June to September 2018. Information on use and consumption of ARVs, compliance with guidelines, change of therapeutic combination as well as their reasons were consulted for the present study. Results: 507 files of PLHIV were collected in the various ATCs. 274 (54.1\%) were from female patients. The most represented age group was 26 to 35 years with 192 patients $(37.9 \%)$. The mean duration of first-line treatment for all patients included was $16.30 \pm$ 5.85 months. The most widely used combination of ARVs overall was TDF +
\end{abstract}


$3 \mathrm{TC}+\mathrm{EFV}$ at $45.4 \% .305$ (60.2\%) PLHIV kept the same first-line treatment molecule throughout the treatment period with an average treatment duration of $12.9 \pm 2.77$ months. The most common combination found in this population was TDF + 3TC + EFV (69.2\%). 202 (39.8\%) PLHIV changed treatment molecule yet respecting the first-line combinations. The average duration of treatment for those who changed molecules was $21.43 \pm 7.25$ months. Before the change, 112 (55.5\%) of these patients were using the ZDV + 3TC + EFV combination. After switching, 105 (52\%) of the patients used the TDF + 3TC + NVP combination. The first reason for changing molecules was its unavailability (53.3\%) in the ATCs. Conclusion: Although some banned molecules are still available in some treatment centers, the guidelines on first-line treatments are respected in different centers in Kinshasa.

\section{Keywords}

Rational Use, ARVs, Dolutegravir, Kinshasa

\section{Introduction}

The Human Immunodeficiency Virus (HIV) is a retrovirus that infects humans, and is responsible for Acquired ImmunoDeficiency Syndrome (AIDS), which is a weakened condition of the immune system making it vulnerable to multiple opportunistic infections.

AntiRetroVirals (ARVs) are molecules used to fight HIV infection. The ultimate goal of AntiRetroViral Treatments (ART) is to achieve complete immune restoration and lasting viral suppression in the infected patient. To ensure efficacy, safety and accessibility, antiretroviral drugs are prescribed according to national guidelines. Nowadays, there are several ARV molecules that have different targets in the life cycle of the virus. They work specifically by inhibiting viral replication and, therefore, slowing the progression of infection. With the arrival of new molecules, the management of ARVs is evolving with the various recommendations of the World Health Organization (WHO) [1].

Rational use of drugs implies that patients receive drugs that are appropriate for their clinical condition, in doses that suit their individual needs, for an adequate period of time, and at the lowest cost for themselves and their communities [2]. Irrational drug use is usually the result of inadequate prescription, and can cause or increase adverse events leading to other illnesses or even death [2]. On the other hand, it is important that the extent and severity of these adverse events be established with a view to either reorienting the management policy or communicating them to patients to improve treatment adherence. The principle of essential drugs stipulates that, at the national level, the choice of a drug line must take into account certain criteria such as the effectiveness of the drug, the inherent risks as well as the needs of the population [3].

In the Democratic Republic of Congo (DRC), since the introduction of ARVs, 
Reverse Transcriptase Inhibitors (RTI) as well as those of viral Protease (PI) are widely used despite the various changes in care policies [4]. Previously, in DRC, the preferred scheme was a combination of 2 Nucleoside Reverse Transcriptase Inhibitors (NRTI-Lamivudine/3TC and Zidovudine/ZDV) and 1 Non-Nucleoside Reverse Transcriptase Inhibitor (NNRTI-Efavirenz/EFV or Nevirapine/NVP) [5]. In the 2017 revision, the new preferred treatment proposed was a combination of 1 NRTI (3TC), 1 Nucleotide Reverse Transcriptase Inhibitor (nRTI-Tenofovir/TDF) and 1 INNRT (EFV) [4]. Until the transition to Dolutegravir, the alternative regimen consisted of 2 NRTI (CBV = 3TC + ZDV) and 1 INNRT (NVP) [4]. This new proposed treatment had the advantage of diversifying the targets of TARV by introducing a nRTI into the preferential treatment. In all cases, respect and observance of these treatment guidelines have always been essential for the success of ART access programs. All changes in treatment regimen done in the past were motivated by WHO recommendations. However, they have never been evaluated by the national program in order to present the adherence to the treatment nor the achievement.

In 2019, the national program adopted a transition plan to introduce Dolutegravir (DTG) into the preferential first-line ART scheme for the DRC [6]. Dolutegravir (DTG) is an ARV of the Integrase Inhibitor (II) class recommended by WHO to improve the management of People Living with HIV (PLHIV) [1]. This new recommendation introduces a molecule with a different target, thus diversifying TARV.

The objective of this study was to contribute to improving the care of People Living with HIV (PLHIV) by reporting on the rational use of Antiretrovirals in patients treated in different Ambulatory Treatment Centers (ATCs) for HIV infection in Kinshasa before the use of Dolutegravir in the national program. This will allow health programs to predict its proper use and make necessary arrangements.

\section{Methods}

\subsection{Type of Study}

The present work is a descriptive cross-sectional study to assess the rational use of first-line Antiretrovirals (ARVs) in People Living with HIV (PLHIV) in various Ambulatory Treatment Centers (ATCs) in Kinshasa before the introduction of Dolutegravir.

\subsection{Population and Study Periods}

Patient records were randomly and rationally selected from 12 different ATCs for HIV in Kinshasa according to three centers per district in the period from June to September 2018. Only complete records of patients on first-line treatment who had come for consultation during this period were consulted for the present study. Therefore, all files which were properly kept at the ATCs during the period of the study were considered.

All the files of patients who had been on ART for more than 6 months, who 
had regular consultations in the different ACTs, who had come for consultation during the period of the study without no discrimination were included. The algorithm presented on the included Figure 1 was used to decide on inclusion.

\subsection{Parameters of Interest}

Information on the use and consumption of ARVs, compliance with guidelines, change of therapeutic combination as well as their reasons were collected for this study.

\subsection{Data Collection and Analysis Methods}

Patient data on $1^{\text {st }}$ line treatment were collected on a collection sheet and then transcribed into an Excel spreadsheet. Quantitative data was presented as absolute value and percentage, and others as mean \pm standard deviation.

\section{Results}

Five hundred and seven (507) files of PLHIV were collected in the different ATCs. Two hundred and seventy-four (274) files, or $54.1 \%$, belonged to female patients and 233, or $45.9 \%$, to male patients. The most represented age group was that of 26 to 35 years with 192 patients (37.9\%), followed by that of 18 to 25 years (25.0\%), that of 36 to 45 years $(21.3 \%)$ and those aged 46 to 55 years (12.2\%). The mean duration of first-line treatment, before the transition, for all patients included in labor was $16.30 \pm 5.85$ months.

The most widely used combination of first-line molecules overall is that of Tenofovir (TDF) + Lamivudine (3TC) + Efavirenz (EFV) at $45.4 \%$ followed by combinations of TDF + 3TC + Nevirapine (NVP) at 20.7\%, TDF + Emtricitabine $($ FTC $)+$ EFV at $14.6 \%$, TDF + FTC + NVP et $11.6 \%$, Stavudine $(\mathrm{d} 4 \mathrm{~T})+3 \mathrm{TC}+$ $\mathrm{NVP}$ at $5.1 \%$ and Zidovudine (ZDV) $+3 \mathrm{TC}+\mathrm{EFV}$ at $2.6 \% \mathrm{EFV}$. All of them are first line combinations prescribed by the national program before the transition.

Three hundred and five (305) PLHIV, or 60.2\%, have not switched to first-line ART. The average duration of treatment for those who did not change molecules

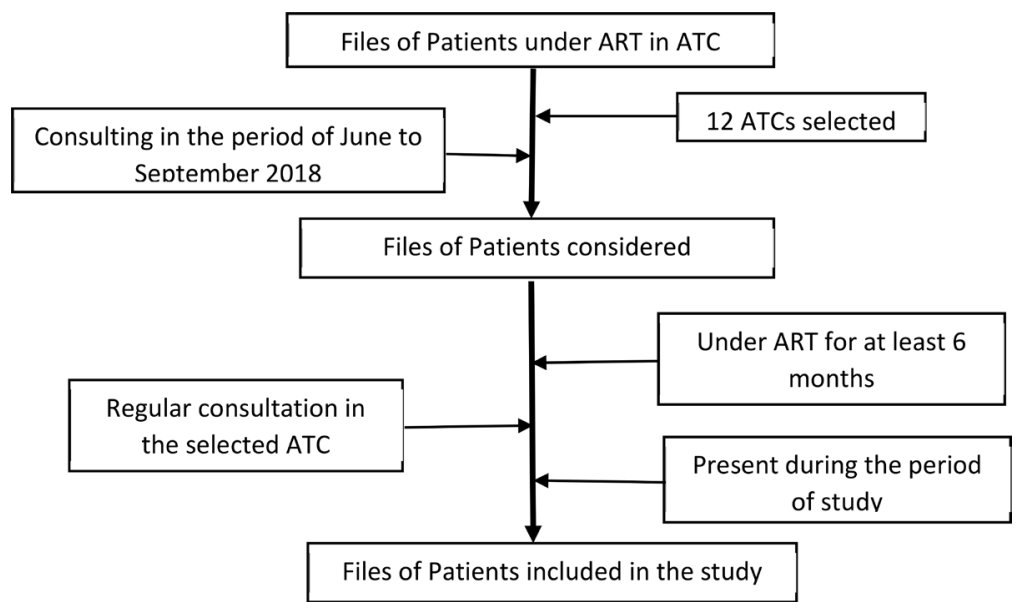

Figure 1. Algorithm used for selection of files. 
was $12.9 \pm 2.77$ months. One hundred and sixty-seven $(54.75 \%)$ records in this group were female patients while 138 (45.25\%) were male patient records. The age group most represented in this group was that of 26 to 35 years old with 123 patients $(40.3 \%)$, followed by that of 18 to 25 years $(30.5 \%)$ and that of 36 to 45 years (19.3\%).

For this group, the most common combination found in this population was $\mathrm{TDF}+3 \mathrm{TC}+\mathrm{EFV}$ (69.2\%), followed by TDF + FTC + EFV (22.6\%), ZDV + $3 \mathrm{TC}+\mathrm{EFV}(4.3 \%), \mathrm{d} 4 \mathrm{~T}+3 \mathrm{TC}+\mathrm{NVP}(2.6 \%)$ and TDF + FTC + NVP (1.3\%).

Two hundred and two (202) PLHIV, or 39.8\%, changed one or two treatment molecules, always keeping the first-line combinations. The average duration of treatment for those who changed molecules was $21.43 \pm 7.25$ months. One hundred and seven (53.0\%) records in this group were female patients while 95 $(47.0 \%)$ were male patient charts. The age group most represented in this group was that of 26 to 35 years with 69 patients (34.1\%), followed by that of 36 to 45 years $(24.3 \%)$ and that of 46 to 55 years (18.8\%).

In this second group, before changing molecules, $55.5 \%$ of these patients used the ZDV + 3TC + EFV combination, $24.7 \%$ used the TDF + FTC + EFV combination, $16.8 \%$ used the TDF $+3 \mathrm{TC}+\mathrm{EFV}$ combination, $2.5 \%$ used the $\mathrm{d} 4 \mathrm{~T}+$ $3 \mathrm{TC}+\mathrm{NVP}$ combination, and $0.5 \%$ used the TDF + FTC + NVP combination. After changing molecules, $52 \%$ of patients used the TDF $+3 \mathrm{TC}+\mathrm{NVP}$ combination, 27.2\% used the TDF + FTC + NVP combination, 9.4\% used the TDF + $3 \mathrm{TC}+$ EFV combination, $8.9 \%$ used the $\mathrm{d} 4 \mathrm{~T}+3 \mathrm{TC}+\mathrm{NVP}$ combination, and $2.5 \%$ used the TDF + FTC + EFV combination.

Table 1 included in the manuscript present all the above state results. The first reason for changing molecules was its availability (53.3\%) in the ATCs, followed by the adverse effects declared by the patient $(28.5 \%)$ and the decisions taken by the prescriber after consultation (18.2\%).

\section{Discussion}

The objective of this study was to assess the rational use of Antiretrovirals in People Living with HIV under first-line treatment treated in different Ambulatory Treatment Centers (ATCs) in Kinshasa before the use of Dolutegravir in the national program.

Five hundred and seven files of PLHIV were collected in the various ATCs. Two hundred and seventy-four records (274), 54.1\%, belonged to female patients and $233,45.9 \%$, to male patients. The sex ratio in favor of women is very common in the setting in the context of HIV infection; several studies present female patients more present than men [7] [8] [9]. The most represented age group was that of 26 to 35 years with 192 patients (37.9\%), followed by that of 18 to 25 years $(25.0 \%)$, that of 36 to 45 years $(21.3 \%)$ and those aged 46 to 55 years (12.2\%). These results are similar to those presented by various studies in our community where the age group most affected by the HIV epidemic is between 18 and 49 years [7] [8] [9]. The mean duration of first-line treatment for all pa- 
tients in labor, before transition, was $16.30 \pm 5.85$ months on ART. This corroborates data from previous studies showing patients followed for $35 \pm 8$ months on first-line ART before switching to a higher line [10].

The most widely used combination of molecules is that of Tenofovir (TDF) + Lamivudine (3TC) + Efavirenz (EFV), TLE, at $45.4 \%$ followed by combinations of TDF + 3TC + Nevirapine (NVP), TLN, at 20.7\%, TDF + Emtricitabine (FTC) + EFV, ART, at 14.6\%, TDF + FTC + NVP at 11.6\%, Stavudine $(\mathrm{d} 4 \mathrm{~T})+3 \mathrm{TC}+$ NVP, TRI, at 5.1\%, and Zidovudine $(Z D V)+3 \mathrm{TC}+\mathrm{EFV}$ at $2.6 \%$. The national recommendations are more or less respected in the different structures, however, the use of Stavudine puts many questions on the table. This molecule has

Table 1. Characteristics of the population.

\begin{tabular}{|c|c|c|c|}
\hline Characteristics & $\begin{array}{l}\text { Patients who did not switch } \\
\text { treatment }(\mathrm{n}=305)\end{array}$ & $\begin{array}{l}\text { Patients who switched } \\
\text { treatment }(n=202)\end{array}$ & Total $(n=507)$ \\
\hline \multicolumn{4}{|l|}{ Sex } \\
\hline Male & $138(45.25 \%)$ & $95(47.0 \%)$ & $233(45.9 \%)$ \\
\hline Female & 167 (54.75\%) & $107(53.0 \%)$ & $274(54.1 \%)$ \\
\hline \multicolumn{4}{|l|}{ Age Interval (years) } \\
\hline $18-25$ & $93(30.5 \%)$ & $34(16.8 \%)$ & $127(25.0 \%)$ \\
\hline $26-35$ & $123(40.3 \%)$ & $69(34.1 \%)$ & $192(37.9 \%)$ \\
\hline $36-45$ & $59(19.3 \%)$ & $49(24.3 \%)$ & $108(21.3 \%)$ \\
\hline $46-55$ & $24(7.9 \%)$ & $38(18.8 \%)$ & $62(12.2 \%)$ \\
\hline $56-65$ & $5(1.6 \%)$ & $11(5.5 \%)$ & $16(3.2 \%)$ \\
\hline+65 & $1(0.4 \%)$ & $1(0.5 \%)$ & $2(0.4 \%)$ \\
\hline $\begin{array}{c}\text { Average duration } \\
\text { of Treatment }\end{array}$ & $12.9 \pm 2.77$ months & $21.43 \pm 7.25$ months & $16.30 \pm 5.85$ months \\
\hline \multicolumn{4}{|l|}{ Treatment } \\
\hline $\mathrm{TDF}+3 \mathrm{TC}+\mathrm{EFV}$ & $211(69.2 \%)$ & $19(9.4 \%)$ & $230(45.4 \%)$ \\
\hline $\mathrm{TDF}+\mathrm{FTC}+\mathrm{EFV}$ & $69(22.6 \%)$ & $5(2.5 \%)$ & $74(14.6 \%)$ \\
\hline $\mathrm{ZDV}+3 \mathrm{TC}+\mathrm{EFV}$ & $13(4.3 \%)$ & 0 & $13(2.6 \%)$ \\
\hline $\mathrm{TDF}+\mathrm{FTC}+\mathrm{NVP}$ & $4(1.3 \%)$ & $55(27.2 \%)$ & $59(11.6 \%)$ \\
\hline $\mathrm{TDF}+3 \mathrm{TC}+\mathrm{NVP}$ & 0 & $105(52.0 \%)$ & $105(20.7 \%)$ \\
\hline $\mathrm{d} 4 \mathrm{~T}+3 \mathrm{TC}+\mathrm{NVP}$ & $8(2.6 \%)$ & $18(8.9 \%)$ & $26(5.1 \%)$ \\
\hline \multicolumn{4}{|l|}{ Previous Treatment } \\
\hline $\mathrm{TDF}+3 \mathrm{TC}+\mathrm{EFV}$ & -- & $34(16.8 \%)$ & $34(16.8 \%)$ \\
\hline $\mathrm{TDF}+\mathrm{FTC}+\mathrm{EFV}$ & -- & $50(24.7 \%)$ & $50(24.7 \%)$ \\
\hline $\mathrm{ZDV}+3 \mathrm{TC}+\mathrm{EFV}$ & -- & $112(55.5 \%)$ & $112(55.5 \%)$ \\
\hline $\mathrm{TDF}+\mathrm{FTC}+\mathrm{NVP}$ & -- & $1(0.5 \%)$ & $1(0.5 \%)$ \\
\hline $\mathrm{TDF}+3 \mathrm{TC}+\mathrm{NVP}$ & -- & 0 & 0 \\
\hline $\mathrm{d} 4 \mathrm{~T}+3 \mathrm{TC}+\mathrm{NVP}$ & -- & $5(2.5 \%)$ & $5(2.5 \%)$ \\
\hline
\end{tabular}


been officially withdrawn since 2013 [5]. This molecule was banned by the WHO following the various reported adverse effects [6].

Three hundred and five (60.2\%) PLHIV kept the same first-line treatment molecule. The average duration of treatment for those who did not change molecules was $12.9 \pm 2.77$ months. One hundred and sixty-seven (54.75\%) records in this group were female patients while 138 (45.25\%) were male patient records. The age group most represented in this group was that of 26 to 35 years with 123 patients (40.3\%), followed by that of 18 to 25 years (30.5\%) and that of 36 to 45 years (19.3\%). The most common combination found in this population was TDF + 3TC + EFV, TLE (69.2\%), followed by TDF + FTC + EFV, ART (22.6\%), $\mathrm{ZDV}+3 \mathrm{TC}+\mathrm{EFV}(4.3 \%), \mathrm{d} 4 \mathrm{~T}+3 \mathrm{TC}+\mathrm{NVP}, \mathrm{TRI}(2.6 \%)$ and TDF + FTC + NVP (1.3\%). These combinations comply with the latest national recommendations on the prescription of first-line ARVs [4] [6].

Two hundred and two (39.8\%) PLHIV changed treatment molecules, always keeping the first-line combinations. The average duration of treatment for those who changed molecules was $21.43 \pm 7.25$ months. Longer exposure to ART is always likely to cause resistance to treatments which often lead to changes in molecules [6] [7]. One hundred and seven (53.0\%) records in this group were female patients while 95 (47.0\%) were male patient charts. In accordance with the literature, the age group most represented in this group was that of 26 to 35 years with 69 patients $(34.1 \%)$, followed by that of 36 to 45 years $(24.3 \%)$ and that of 46 to 55 years (18.8\%). In this group, before changing molecules, 55.5\% of patients used the ZDV + 3TC + EFV combination, $24.7 \%$ used the TDF + FTC + EFV combination, $16.8 \%$ used the TDF + 3TC + EFV combination, 2.5\% used the $\mathrm{d} 4 \mathrm{~T}+3 \mathrm{TC}+\mathrm{NVP}$ combination, and $0.5 \%$ used the TDF + FTC + NVP combination. These combinations are no longer up to date on the latest recommendations, they are combinations of previous recommendations [5]. After changing molecules, $52 \%$ of patients used the TDF $+3 \mathrm{TC}+\mathrm{NVP}$ combination, $27.2 \%$ used the TDF + FTC + NVP combination, 9.4\% used the TDF + 3TC + EFV combination, $8.9 \%$ used the $\mathrm{d} 4 \mathrm{~T}+3 \mathrm{TC}+\mathrm{NVP}$ combination, and $2.5 \%$ used the TDF + FTC + EFV combination. The combinations after switching are closer to current treatment recommendations [4] [6]. Hence the regular change of ART guidelines may be the cause of non-compliance with these recommendations.

Nevertheless for this group, according to the data, the first reason for changing molecules was its availability (53.3\%) in the ATCs, followed by adverse effects in the patient (28.5\%) and decisions taken by the prescriber (18.2\%). This is justified because the supply is made by the national programs which adapt their stocks according to the decisions to change molecules.

\section{Limitations of the Study}

This is a descriptive cross-sectional study based on patients' files and records. Therefore, only available patients' data have been collected to be presented. The type and the restriction of the study did not allow to record additional informa- 
tion such as clinical and biological data of patients. Only available data on patients files could be presented.

\section{Conclusion}

Although some banned molecules are still available in some treatment centers, the guidelines on first-line treatments are respected in the Kinshasa treatment centers.

\section{Conflicts of Interest}

The authors declare no conflicts of interest regarding the publication of this paper.

\section{References}

[1] World Health Organization (2019) Update of Recommendations on First- and Second-line Antiretroviral Regimens: HIV Treatment. Policy Brief.

[2] World Health Organization. https://www.who.int/medicines/areas/rational use/fr/

[3] Immita Cornaz. Les médicaments essentiels. https://journals.openedition.org/aspd/1060

[4] Programme National de Lutte contre le VIH/SIDA et les IST (PNLS). Ministère de la Santé Publique, République Démocratique du Congo (2017) Guide National de Prise en Charge.

[5] Programme National de Lutte contre le VIH/SIDA et les IST (PNLS). Ministère de la Santé Publique, République Démocratique du Congo (2013) Guide National de Prise en Charge.

[6] Programme National de Lutte contre le VIH/SIDA et les IST (PNLS). Ministère de la Santé Publique, République Démocratique du Congo (2019) Révision du Guide National de Prise en Charge.

[7] Kamangu, N.E., Wumba, D.R., Situakibanza, N.T.H., Lukusa, T.P., Kapend, K.L., Mvumbi, L.G., Hayette, M.P. and Kalala, L.R. (2018) Molecular Epidemiology of Human Immunodeficiency Virus Type 1 and Therapeutic Monitoring of Patients Treated in Kinshasa/Democratic Republic of the Congo. International Journal of HIV and AIDS Research, 1, 6-11

[8] Bongenya, I.B., Bukongo, N.R., Bulanda, I.B., Chuga, D., Kabasele, D.J.Y., Okonda, O.M., Tshisumbu, C. and Kamangu, N.E. (2018) Gender Difference in Early Treatment Patients for Human Immunodeficiency Virus Type 1 Infection in Kinshasa, Democratic Republic of Congo. Mathews Journal of HIVI AIDS, 3, 18.

[9] Kamangu, N.E., Bulanda, I.B., Bongenia, I.B., Botomwito, T.H., Mvumbi, L.G., De Mol, P., Vaira, D., Hayette, M.-P. and Kalala, L.R. (2015) Virological Profile of Patients Infected with HIV Starting Antiretroviral Treatment in Kinshasa. Open Access Library Journal, 2, Article ID: e1564. https://doi.org/10.4236/oalib.1101564

[10] Kamangu, N.E., Bwiri, B.B. and Mvumbi, G.L. (2019) Clinical and Paraclinical Profile of People Living with Human Immunodeficiency Virus on Second Line Treatment in Kinshasa, Democratic Republic of Congo. Open Access Library Journal, 6, Article ID: e5499. https://doi.org/10.4236/oalib.1105499 


\section{Abbreviations and Acronyms}

3TC: Lamivudine

ART: AntiRetroViral Treatment

ART': TDF + FTC + EFV

ARV: Antiretroviral

ATC: Ambulatory Treatment Center

CBV: ZDV + 3TC

d4T: Stavudine

DRC: Democratic Republic of Congo

DTG: Dolutegravir

EFV: Efavirenz

FTC: Emtricitabine

HIV: Human Immunodeficiency Virus

II: Inhibitor of Integrase

NVP: Nevirapine

NNRTI: Non-Nucleoside Reverse Transcriptase Inhibitor

nRTI: Nucleotide Reverse Transcriptase Inhibitor

NRTI: Nucleoside Reverse Transcriptase Inhibitor

PI: Protease Inhibitor

PLHIV: Person Living With HIV

RTI: Reverse Transcriptase Inhibitor

TDF: Tenofovir

TLE: TDF + 3TC + EFV

TRI: $\mathrm{d} 4 \mathrm{~T}+3 \mathrm{TC}+\mathrm{NVP}$

TRV: TDF + FTC

WHO: World Health Organization

ZDV: Zidovudine. 